\title{
The Intriguing Life of Massive Galaxies: Introducing the Final Discussion
}

\author{
Alvio Renzini ${ }^{1}$ \\ ${ }^{1}$ INAF - Osservatorio Astronomico di Padova \\ email: alvio.renzini@oapd.inaf.it
}

\begin{abstract}
This is a brief introduction to the closing discussion of the IAU Symposium 295, "The Intriguing Life of Massive Galaxies", that was held in Beijing from August 27 through 31, 2012. The discussion was focused on only four hot items, namely 1) the redshift evolution of the size of passively evolving galaxies, 2) the evolution with redshift of the specific star formation rate, 3) quenching of star formation in galaxies and dry merging, and 4) the IMF.
\end{abstract}

Keywords. Galaxy Evolution; Elliptical Galaxies; Star Formation; IMF

\section{Introduction}

I have been asked by the organizers to introduce the final discussion of this IAU Symposium 295 "The Intriguing Life of Massive Galaxies" thus trying to provoke a wide participation by the audience. I have chosen to focus the discussion on just a few among the many issues that have been addressed and debated at this exciting meeting. For each topic, after five minutes of introduction, some ten or more minutes of general discussion followed. All this was recorded but at this point it is not clear whether it will become accessible on line on the site of the symposium. Therefore, this short article is limited to a report of my introduction to each topic, in the wish of attracting readers towards the actual event, if the records will become available.

I wish to start by confessing that already quite some time ago I lost faith in the ability of theoretical models to tell us how real galaxies form and evolve, being them of the semianalytic or the hydro-simulation variety. The reason is that once the mess of baryonic physics is added to the clean elegance of dark matter N-body realizations most -if not all- predictive power is lost in a plethora of adjustable parameters meant to describe the many physical processes at work. Just to name a few: star formation, galactic winds, cold streams, supermassive black hole formation, nuclear activity and its feedback, chemical evolution, galaxy mergers, starbursts, disk instabilities, multiphase ISM, supernova feedback, ram pressure stripping, dust formation, radiative transfer and many more. For example, Benson \& Bower (2010) list over two dozens adjustable parameter for their semi-analytic model. So, being an infidel, my view of theory would be biased and I preferred to drive the discussion towards some specific points that direct observations may help to clarify.

\section{The Size Evolution of Passively Evolving Galaxies}

Over a dozen talks have been dealing with the size of passively evolving galaxies (PEG) at the various redshifts. Sizes are straightforward to visualize and effective radii $\left(R_{\mathrm{e}}\right)$ are relatively easy to measure, thus over one hundred papers have been dedicated in recent years to report on how high redshift PEGs are smaller and denser at given stellar mass compared to their local analogs, and what processes may account for their apparent 
growth by up to a factor of $\sim 4$ from $z \sim 2.5$ to $z \sim 0$. Indeed, at fixed stellar mass the average $R_{\mathrm{e}}$ of PEGs ( $\left.<R_{\mathrm{e}}\right\rangle$, as normalized to the local $R_{\mathrm{e}}-M_{*}$ relation) steadily declines with increasing redshift, a trend that -with few exceptions (e.g., Valentinuzzi et al. 2010; Cassata et al. 2011; Newman et al. 2012)- has been often entirely ascribed to the physical growth of individual galaxies. Accretion of an envelope of small satellites (minor mergers) has been widely entertained as the leading mechanism to puff up the individual high- $z$ compact PEGs until they reach their due, final dimension. Yet, this is only part of the story, and likely only a minor one.

The fact is that the comoving number density of massive PEGs is increasing by a large factor $(\sim 25)$ between $z=2.5$ and 0 , with most of this increase taking place between $z=2.5$ and $\sim 1$. Hence, the run of the average size of PEGs primarily reflects the size with which at each redshifts they first appear as quenched, rather than the subsequent grow of each of them. Thus, the real challenge is to understand why galaxies which are quenched at later times are born bigger than those formed earlier, rather than (or not only) to understand to which extent individual PEGs secularly increase their size. Precursors to PEGs must be star forming galaxies likely spending most of their time on their main sequence growing inside-out. Hence, the later they are quenched the bigger they are, and presumably the bigger their passive remnant. Is just this the main story?

An intriguing aspect is that the number density of compact PEGs actually appears to increase with cosmic time, peaking at $z \sim 1$ (e.g., Cassata et al. 2011), right when $\left.<R_{\mathrm{e}}\right\rangle$ is most rapidly increasing (!), and then starts to drop at lower redshifts. Thus, between $z \sim 2.5$ and $\sim 1$ the distribution of effective radii of newly formed PEGs is rapidly evolving, and does so in such a way that the birth rate of normal size PEGs is higher than a sill increasing birth rate of compact ones. It is only below $z \sim 1$ that the number density of compact PEGs starts to drop, and some growth of individual galaxies must take place. Thus, understanding the redshift evolution of $\left\langle R_{\mathrm{e}}>\right.$ requires to follow simultaneously the complex interplay between the growth of individual galaxies and the birth of new PEGs and their size distribution. A key issue that remains to be settled concerns the number density of compact PEGs in the local Universe: how does it compare with the number density at redshift 1 or 2 ?

\section{The Specific Star Formation Rate}

The specific star formation rate (sSFR) of main sequence galaxies as a function stellar mass and cosmic time, conveniently parameterized as $\operatorname{sSFR}\left(M_{*}, t\right) \propto M_{*}^{\beta} t^{-\gamma}$, plays a pivotal role in galaxy evolution. It controls the growth rate of galaxies, the evolution of their mass function, and can have a direct effect even on quenching of star formation itself. Despite its importance, the precise value of $\beta$ is still uncertain, with values in the literature spanning a very wide range $(-0.4 \lesssim \beta \lesssim 0)$, the result depending on how star forming galaxies are selected, and how SFR and $M_{*}$ are measured.

There is clear evidence that the sSFR drops systematically with time since $z \sim 2$, by at least a factor of $\sim 20$, hence $\gamma \simeq 2.2-2.5$. This drop with time (increase with lookback time and redshift) runs almost parallel to the specific mass increase rate of merging dark matter halos, thus hinting for a direct link between star formation rate and accretion rate of the haloes. A simple interpretation of such quasi-parallelism is that the supply of fresh baryons (gas) feeding star formation in galaxies follows the dark matter halo accretion rate, as if baryons and dark matter were fairly bound to each other. However, beyond redshift $\sim 2$ the sSFR appears to flatten, then remaining nearly constant (i.e., $\gamma \simeq 0$ ) all the way to very high redshifts (e.g., Gonzalez et al. 2010). On the contrary, the specific increase rate of dark matter haloes keeps increasing. 
This divergence from parallelism beyond $z \sim 2$ has caused some concern on the theoretical side (e.g., Weinmann, Neinstein \& Dekel 2011), as it would apparently demand drastic modifications to widely adopted assumptions, as if baryons and dark matter were substantially decoupled at early cosmic times. Thus, measuring the actual value of $\gamma$ for redshifts beyond $\sim 2.5$ has potentially strong implications for the relative behavior of baryons and dark matter. It may also be especially relevant for reionization, but may be relatively unimportant for the actual mass growth of galaxies, which most takes place at lower redshifts. In phenomenological models of galaxy evolution (e.g., Peng et al. 2010), compared to a $\gamma=0$ case a $\gamma>0$ beyond $z \sim 2.5$ would imply a different ratio of the final galaxy stellar mass to the mass of the initial seed, having otherwise rather irrelevant effect on the final outcome. But a precise measurement of the run of the sSFR with mass and time (i.e., of $\beta$ and $\gamma$ ) remains a central issue for a proper understanding of galaxy formation and evolution. Contrary to previous results, it has been reported at this meeting that the sSFR may still be increasing somewhat even well beyond $z \sim 2$ (e.g., Stark et al. 2013). Thus, the issue remains unsettled and worth attracting further studies.

\section{Quenching and Dry Merging}

Quenching of star formation then turning to passive evolution is perhaps the most salient event in the life of a galaxy. Observations tell us that the fraction of quenched galaxies is a strong function of both stellar mass and local overdensity. Thus, two distinct physical processes must exists, dubbed mass quenching and environment quenching, that act independent of each other (Peng et al. 2010). What remains to be established is the physical nature of these two processes: what is environment quenching? and, what is mass quenching?

Is environment quenching just ram pressure stripping? or strangulation? or a combination thereof? Is mass quenching a process internal to galaxies themselves, such as e.g., AGN feedback? or is it an external process, also related to the environment? (e.g., galaxies are quenched when the mass of the host dark matter halo exceeds a threshold value). Does mass quenching work by ejecting gas out of galaxies or by preventing accretion of cold gas into galaxies? In other words, what is mass in mass quenching? Is it $M_{*}$ or $M_{\mathrm{h}}$ ? Theoreticians appear to be equally divided, and probably only observations can answer the question.

Yet, quenching may not be the last event in the evolution of massive galaxies. Postquenching (dry) merging can also take place, and in a popular cartoon such further mass increase may be as large as a factor $\sim 10$ (see Figure 1). This scenario was motivated by the perception that star-forming (SF) galaxies as massive as the most massive quenched galaxies would not exist, hence massive PEGs $\left(M_{*} \gtrsim 10^{11} M_{\odot}\right)$ could not form by just quenching star formation in a blue cloud galaxy, then moved to the red sequence, i.e., there would be not enough massive blue galaxies in the distant Universe with masses comparable to those of the brightest red galaxies. Actually, massive enough SF galaxies do exist, but they are very rare. The reason why they are rare is precisely because they are growing in mass so rapidly that their life expectancy (as star forming) is very short: they soon are going to be mass quenched! Thus, the cartoon is missing the main point: most of the action, i.e., most of the mass quenching, takes place near the top end of the mass function of SF galaxies, for $M_{*} \gtrsim 10^{11} M_{\odot}$, within the open ellipse in Figure 1, a region left empty in the original plot.

Now, dry merging does certainly exist, but its role is not as prominent as sometimes envisaged. In high density regions the Schechter $M_{*}^{*}$ of quenched galaxies is just $\sim 0.1$ dex higher than in low density regions, where merging is almost absent (Peng et al. 


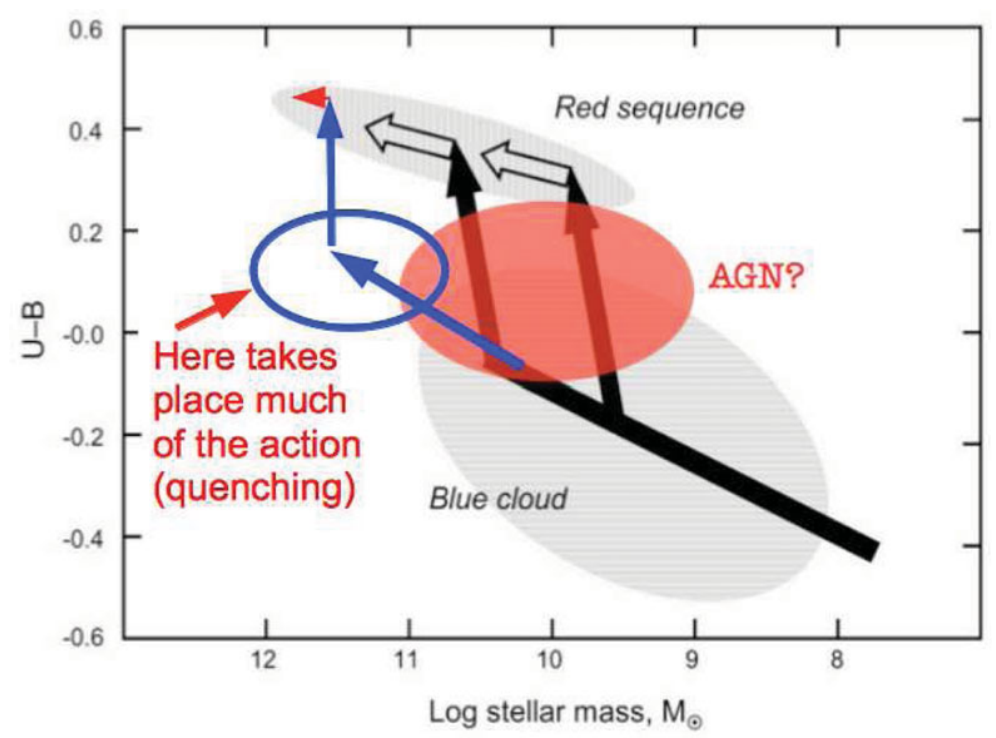

Figure 1. A modification of the original cartoon with an idealized sketch of the transition from star-forming "blue cloud" galaxies to quenched "red sequence" galaxies (black and grey scale, Faber et al. 2007). In color the actual path of galaxies being mass quenched is indicated (in blue), together with a modest further mass increase by dry merging (short red arrow).

2010). Thus, dry mergers make just an average $\sim 20 \%$ mass increase, certainly not a factor $\sim 10$.

\section{The IMF}

Finally the IMF (usually described by a power law $d N \propto M^{-s} d M$ ). It is currently common practice to adopt a universal IMF for a broad range of astrophysical applications, such as e.g., to estimate stellar masses and star formation rates of galaxies from the local to the most distant Universe so far explored. Yet, it is perfectly legitimate to entertain the notion that the IMF may not be universal. Indeed, from time to time one appeals to different IMFs to ease perceived discrepancies between some theoretical models and observations (e.g., Davé 2008), or between the dynamical and stelllar population mass to light ratios of galaxies (e.g., Cappellari et al. 2012). Thus, sometimes one appeals to a top heavy IMF with a lot of massive stars boosting the luminosity and metal production rate, sometime to a bottom heavy IMF, with a lot of low mass stars, making just mass but little light and no metals. In particular, in a recent surge of papers observational evidence has been presented that would favor a very bottom heavy IMF in massive elliptical galaxies. This was based on the strength of the Na I $\lambda 8190 \AA$ doublet and the FeH Wing-Ford band at $\lambda 9900 \AA$ which both are strong in dwarfs and weak in giants (van Dokkum \& Conroy, 2012; Ferreras et al. 2013; and references therein). These features appear to be stronger in such galaxies than in synthetic stellar population models which adopt a Milky Way (bottom light) IMF, as illustrated in Figure 2 (from van Dokkum \& Conroy 2012).

Notice that the depth of the NaI feature is just a few percent of the (pseudo)continuum, and then a drastic variation of the IMF (with the low-mass slope changing from $\mathrm{s}=1.35$ to 4) leads to a variation of the central line depth from $\sim 96.2 \%$ to just $\sim 95.2 \%$. Formally, as shown in Figure 1, even steeper IMFs would be required for the ellipticals with the highest velocity dispersion. Clearly, the NaI feature is very insensitive to the 


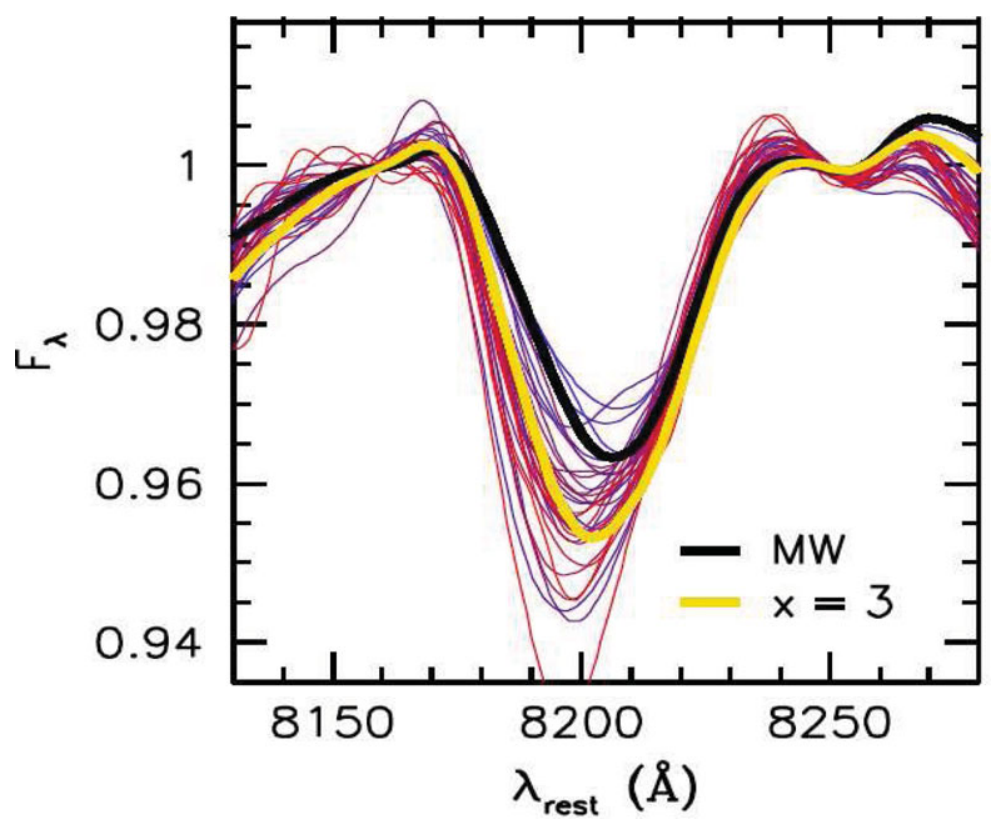

Figure 2. The strength of the NaI $\lambda 8190 \AA$ doublet (here blended by velocity dispersion) in elliptical galaxies, color coded blue to red with increasing velocity dispersion (from van Dokkum \& Conroy 2012). Two synthetic stellar population spectra are also shown for an age of $13 \mathrm{Gyr}$ and Milky Way bottom light IMF $(s=1.35)$ and a bottom heavy IMF $(s=4$ equivalent to a logarithmic slope $x=3$ as $s=x+1)$.

IMF slope and, moreover, deriving such slope from the strength of this feature rests entirely on the reliability of the stellar population models used to draw synthetic spectra such as those shown in Figure 1. Thus, to trust the resulting IMF one has to trust the ability of synthetic models to reproduce the feature with exquisite accuracy. Therefore, a check of such models should be mandatory before taking into serious consideration systematic IMF variations as a function of the velocity dispersion of elliptical galaxies. This is especially true given that models are particularly uncertain (i.e., uncalibrated) for the super-solar metallicities typical of the most massive elliptical galaxies.

Any inference on the IMF from integrated light ultimately rests on synthetic stellar population models and therefore on their reliability. For example, Cappellari et al. (2012) have convincingly demonstrated that mass closely follows light in the core of elliptical galaxies, thus contributing to break the IMF-dark matter degeneracy. Having constrained as marginal the dynamical effect of dark matter, they find that in the core of elliptical galaxies the $M / L$ ratio as inferred from dynamical modeling increases with $M_{*}$ and central velocity dispersion $\sigma$ more than current stellar population models predict. They then interpret this departure in terms of a systematic trend of the IMF with increasing galaxy mass and/or $\sigma$. The $M / L$ ratio is certainly a more fundamental, bulk property of galaxies compared to a weak spectral feature. Yet, also in this case the inference on the IMF is correct only as long as stellar population models are correct. Worth noting is indeed that also metallicity increases with mass and/or $\sigma$ and gets super solar at high mass and $\sigma$ : right where both the heavier IMF is demanded, and where population models are less reliable. There is in fact another degeneracy yet to be broken, one between the IMF and stellar population models, whose use is unavoidable to infer anything about the IMF. 


\section{References}

Benson, A. J. \& Bower, R. 2010, MNRAS, 405, 1573

Cappellari, M. et al. 2012, Nature, 484, 485

Cassata, P., Giavalisco, M., Guo, Y. et al. 2011, ApJ, 743, 96

Davé, R. 2008, MNRAS, 385, 147

Ferreras, I. et al. 2013, MNRAS, 429, 15

Gonzalez, V. et al. 2010, ApJ, 713, 115

Newman, A. B., Ellis, R. S., Bundy, K. et al. 2012, ApJ, 746, 162

Peng, Y. et al. 2010, ApJ, 721, 193

Stark, D. P. et al. 2013, ApJ, 763, 129

Valentinuzzi, T., Poggianti, B. M., Saglia, R. P. et al. 2010, ApJ, 721, L19

van Dokkum, P. \& Conroy, C. 2012, ApJ, 760, 70

Weinmann, S. M., Neinstein, E., \& Dekel, A. 2011, MNRAS, 417, 2737 